OPEN ACCESS

Edited by:

Peilin Jia,

University of Texas Health Science Center, United States

Reviewed by: Xiong $\mathrm{Li}$,

East China Jiaotong University, China

Jialiang Yang,

Geneis (Beijing) Co. Ltd, China

*Correspondence:

Guohua Huang

guohuahhn@163.com

Specialty section:

This article was submitted to

Bioinformatics and

Computational Biology,

a section of the journal

Frontiers in Genetics

Received: 12 September 2019 Accepted: 05 December 2019

Published: 14 February 2020

Citation:

Huang G, Zheng Y, Wu Y-Q, Han G-S and Yu Z-G (2020) An Information

Entropy-Based Approach for Computationally Identifying Histone Lysine Butyrylation.

Front. Genet. 10:1325.

doi: 10.3389/fgene.2019.01325

\section{An Information Entropy-Based Approach for Computationally Identifying Histone Lysine Butyrylation}

\author{
Guohua Huang $^{1 *}$, Yang Zheng ${ }^{1}$, Yao-Qun $\mathrm{Wu}^{1,2}$, Guo-Sheng $\mathrm{Han}^{2}$ and Zu-Guo Yu $\mathrm{u}^{2,3}$ \\ ${ }_{1}^{1}$ Provincial Key Laboratory of Informational Service for Rural Area of Southwestern Hunan, Shaoyang University, Shaoyang, \\ China, ${ }^{2}$ Key Laboratory of Intelligent Computing and Information Processing of Ministry of Education and Hunan Key \\ Laboratory for Computation and Simulation in Science and Engineering, Xiangtan University, Xiangtan, China, ${ }^{3}$ School of \\ Electrical Engineering and Computer Science, Queensland University of Technology, Brisbane, QLD, Australia
}

Butyrylation plays a crucial role in the cellular processes. Due to limit of techniques, it is a challenging task to identify histone butyrylation sites on a large scale. To fill the gap, we propose an approach based on information entropy and machine learning for computationally identifying histone butyrylation sites. The proposed method achieves 0.92 of area under the receiver operating characteristic (ROC) curve over the training set by 3 -fold cross validation and 0.80 over the testing set by independent test. Feature analysis implies that amino acid residues in the down/upstream of butyrylation sites would exhibit specific sequence motif to a certain extent. Functional analysis suggests that histone butyrylation was most possibly associated with four pathways (systemic lupus erythematosus, alcoholism, viral carcinogenesis and transcriptional misregulation in cancer), was involved in binding with other molecules, processes of biosynthesis, assembly, arrangement or disassembly and was located in such complex as consists of DNA, RNA, protein, etc. The proposed method is useful to predict histone butyrylation sites. Analysis of feature and function improves understanding of histone butyrylation and increases knowledge of functions of butyrylated histones.

Keywords: butyrylation, random forest, histone, post-translational modification, information entropy

\section{INTRODUCTION}

Butyrylation, a type of post-translation modification (PTM), refers to a biochemical interaction process where the butyryl functional group covalently modifies the lysine amino acid (Chen et al., 2007; Lu et al., 2018). Protein butyrylation is a newly discovered PTM (Chen et al., 2007). In the past 5 years, butyrylation's roles in the cellular process have been gradually uncovered. For example, Goudarzi et al. (2016) confirmed that histone butyrylation directly stimulates gene expression and inhibits Brdt Binding, Xu et al. (2018) found that butyrylation and acetylation are responsible for the phenotype and metabolic shifts of the endospore-forming Clostridium acetobutylicum, and Lu et al. (2018) revealed that butyrylation prefers poising gene activation by external stresses in the rise of submergence and starvation. Nevertheless, compared to such extensively-studied PTMs as 
acetylation (Kiemer et al., 2005; Basu et al., 2009; Gnad et al., 2010; Choudhary et al., 2014) and methylation (Chen et al., 2006; Shi et al., 2012b; Hamamoto et al., 2015; Shi et al., 2015; Wei et al., 2018), few functions of butyrylation are known. With indepth exploration of butyrylation, more biological functions of butyrylation will undoubtedly be found.

Identifying butyrylation sites is an important foundation to further explore its functions. Biotechnologies whose representative is mass spectrometry provide a necessary approach to identify PTMs including butyrylation. Zhang et al. (2008) found four lysine butyrylation sites in histone yeast, $\mathrm{Xu}$ et al. (2014) 11 histone butyrylation sites in human cells, and Lu et al. (2018) identified four histone butyrylation sites in rice using mass spectrometry. Obviously, this strategy is not only laborintensive and time-consuming, but also generally lowthroughput. On the contrary, bioinformatics approaches provide an alternative to explore PTM sites, with characteristic being high-throughput. Since Hansen et al. (1995; 1998) proposed a method for computationally predicting mucin type O-glycosylation sites in the 1990s, dozens of computational approaches have been developed for identifying PTM sites (Blom et al., 2004; Xue et al., 2006; Zhou et al., 2006; Xu et al., 2008; Xu et al., 2010; Liu et al., 2011; Cai et al., 2012; Shi et al., 2012b; Zhang et al., 2012; Zhao et al., 2012; Xu et al., 2013; Zhang et al., 2013; Zhao et al., 2013; Huang et al., 2014; Shi et al., 2015; $\mathrm{Xu}$ et al., 2015a; Zhou et al., 2016). For instances, glycosylation identification includes the neural network-based method (Hansen et al., 1998), the support vector machine-based method (Li et al., 2006; Chen et al., 2008; Sasaki et al., 2009), the random forest-based method (Hamby and Hirst, 2008; Chuang et al., 2012), and ensemble learning algorithms (Caragea et al., 2007). Features used for predicting methylation sites are from protein sequences (Shao et al., 2009; Zhang et al., 2013; Qiu et al., 2014; Zhang et al., 2015; Wei et al., 2018), structure (Shien et al., 2009) or amino acid properties (Shi et al., 2012a). Xu et al. (2015b) proposed a pseudo amino acid composition-based method for predicting lysine succinylation. Zhou et al. (2004) proposed the GPS method for phosphorylation prediction, and $\mathrm{Xu}$ et al. (2008) proposed the method SUMOpre for sumoylation prediction. These computational methods are capable of screening potential modified sites on a large scale in a little time and help the former methods narrow the scope of verification of it. Here, we didn't plan to comprehensively review and discuss them, but propose a novel method based on information entropy and random forest for predicting histone butyryllysine. To the best of my knowledge, this is the first computational method for predicting butyrylation.

\section{METHOD AND MATERIALS}

\section{Materials}

One hundred butyrylated proteins were retrieved by searching both the Uniprot database (UniProt Consortium, 2018): https:// www.uniprot.org/ and the Protein Lysine Modifications Database (PLMD): http://plmd.biocuckoo.org/ (Xu et al., 2017). The Uniprot database is a comprehensive repository of function annotation and sequences of proteins, which is updated every 2 months. The PLMD is dedicated to specifically collect lysine-modified proteins, and the current version 3.0 contains 284,780 modification events of 20 types of lysine-modified PTMs from 53501 proteins, including butyrylation, crotonylation and propionylation. Searching the Uniprot database with the keyword "butyryllysine", we retrieved 91 butyrylated histones containing 317 butyrylation sites with the manual assertion. We downloaded the butyrylation data from the PLMD. Merging these two datasets and then removing abnormal proteins, we got 100 unique histones. To eliminate dependency of the computational method on homology, it is a general step to remove homology among protein sequences. The computational clustering tool (Huang et al., 2010) was used to cluster these 100 protein sequences with the sequence identity cut-off 0.7. Thirteen representative protein sequences were obtained among which sequence identity of any two is no more than 0.7. We selected six proteins from the Uniprot database as the training set which contained 17 butyrylation sites and the remaining seven from the PLMD as the testing set which contained nine butyrylation sites.

\section{Method}

As shown in Figure 1, the overall workflow of the proposed method consists mainly of four steps: cutting sequence, sequence encoding, training and predicting. The training and the predictive butyrylation histone sequences were cut into fragments which centered lysine with respectively $\mathrm{N}$ amino acid residues in the upstream and the downstream of it. That is, the window of $(2 \mathrm{~N}+1)$ residues centering lysine were separated out. For the windows containing lysine but less than $2 \mathrm{~N}+1$ residues, we prefixed or suffixed the character " $\mathrm{X}$ " to it for complement. The fragments undergoing butyrylation event were viewed as positive samples. We randomly selected 18 non-butyrylation fragments from the training set as training negative samples, and 18 non-butyrylation ones from the testing set as the testing negative samples. The Supplementary Table 1 listed all the training and the testing butyrylation as well as the non-butyrylation sites. For each fragment with $(2 \mathrm{~N}+1)$ resides, the information entropy-based encoding (IEE) and the composition of k-space amino acid pair (CKSAAP) transformed it into numerical feature. After the random forest algorithm trained a classifier using the training set with the numerical features, the unknown protein sequences were input into the trained classifier for final prediction.

\section{IEE}

Histone butyrylation is assumed as a stochastic system described as $\mathrm{P}^{i}(\alpha)$ which stands for probability of the amino acid $\alpha$ occurring at the i-th position. Obviously, $\mathrm{P}^{i}(\alpha)$ is an $m$-by- $n$ matrix where $m$ is the number of characters of amino acid (here $m$ is 21 ) and $n$ the length of the sequence (here $n=2^{\star} \mathrm{N}+1$ ). This stochastic system is measured by the information entropy of 


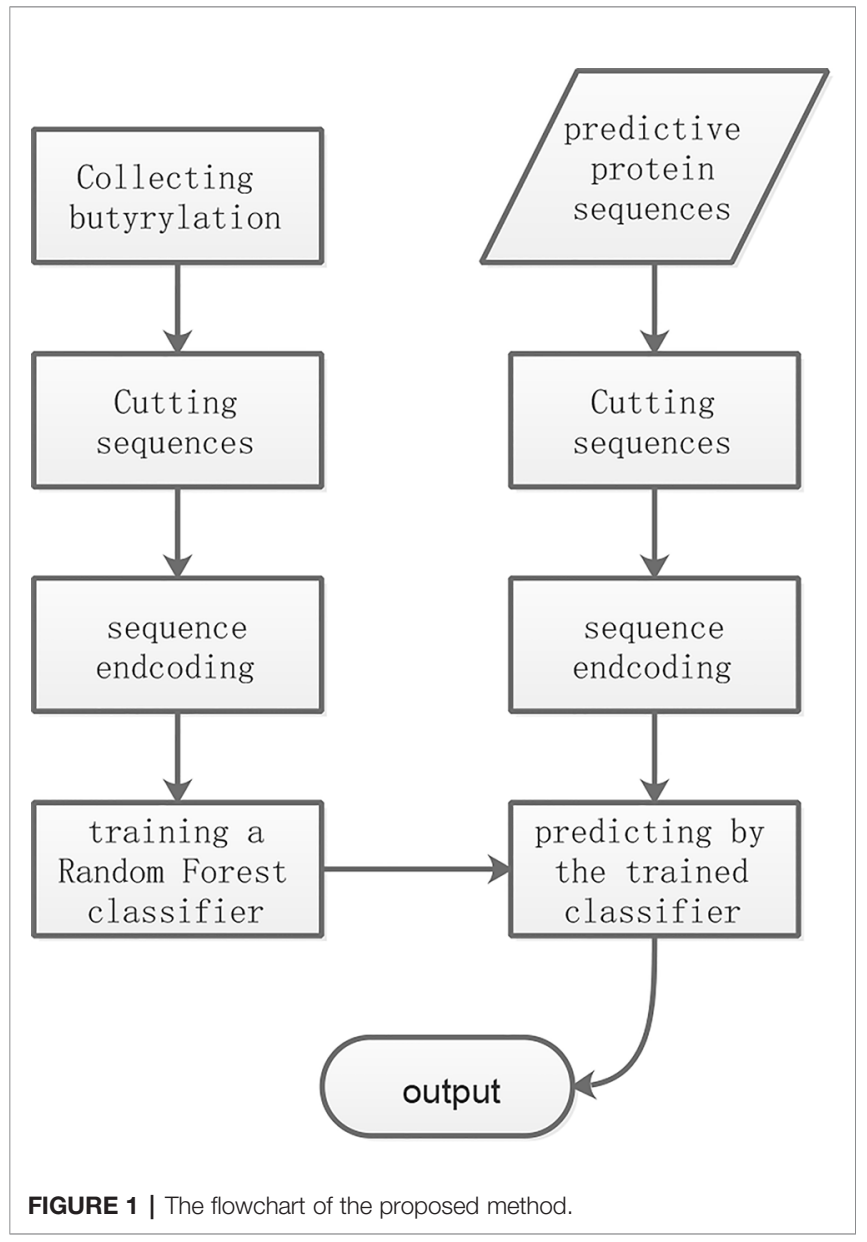

amino acid (PIEA) and the information entropy of position (PIEP), which are denoted respectively by

$$
\operatorname{PIEA}(\alpha)=\sum_{i=1}^{2 N+1}-P^{i}(\alpha) \log P^{i}(\alpha)
$$

and

$$
\operatorname{PIEP}(i)=\sum_{\alpha \in \Phi}-P^{i}(\alpha) \log P^{i}(\alpha)
$$

where $\Phi$ represents the set of characters of amino acid. $\mathrm{P}^{i}(\alpha)$ can be estimated by calculating frequencies of amino acid over all the positive samples in the training set, respectively. The PIEA and the PIEP represent uncertainty of the butyrylation system. The more the PIEA and the PIEP are, the more uncertainty the system is. After a new sample $s$ was added to the system, its information entropies of amino acid and position are denoted by PIEPs and PIEAs. The variation of information entropies after addition of the new sample to the system is defined by

$$
\operatorname{PVIEA}=\operatorname{PIEA}(\alpha)-\operatorname{PIEAs}(\alpha)
$$

and

$$
\operatorname{PVIEP}=\operatorname{PIEP}(\mathrm{i})-\operatorname{PIEPs}(\mathrm{i}) .
$$

Similarly, the non-butyrylation system is also assumed as a distinct stochastic system $\mathrm{N}^{i}(\alpha)$ which is estimated by calculating frequencies of amino acid over all the negative samples in the training set, respectively. The information entropies of amino acid (NIEA) and the information entropies of position (NIEP) for the non-butyrylation system are defined by

$$
\operatorname{NIEA}(\alpha)=\sum_{i=1}^{2 N+1}-\mathrm{N}^{i}(\alpha) \log \mathrm{N}^{i}(\alpha)
$$

and

$$
\operatorname{NIEP}(i)=\sum_{\alpha \in \Phi}-\mathrm{N}^{i}(\alpha) \log N^{i}(\alpha)
$$

The variation of information entropies after addition of the new sample $s$ to the non-butyrylation system is defined by

$$
\operatorname{NVIEA}=\operatorname{NIEA}(\alpha)-\operatorname{NIEAs}(\alpha)
$$

and

$$
\text { NVIEP = NIEP(i) }-\operatorname{NIEPs}(\mathrm{i}),
$$

where NIEAs and NIEPs denote respectively information entropies of amino acid and position after addition of the new sample to the non-butyrylation system. The new sample is encoded by PVIEA-NVIEA and PVIEP-NVIEP. Therefore, for each sample, we obtain $(21+2 \mathrm{~N}+1)$ feature to represent it.

\section{CKSAAP}

The CKSAAP is occurrence frequency of $k$-spaced amino acid pair which is spaced by up to $k$ residues. $k$ is equal to or more than 0 . For example, AA, AC, ..., YX and XX belong to 0 -spaced amino acid pair, while $\mathrm{AA}, \mathrm{AC}, \ldots . . \mathrm{XX}, \mathrm{ABA}, \mathrm{ABC}, \ldots$, and $\mathrm{XBX}$ to 1 -spaced amino acid pair. Generally, there are $(K+1)^{\star} 21^{\star} 21$ features for k-spaced amino acid pair. The CKSAAP were widely applied to prediction of phosphorylation, methylation, palmitoylation, pupylation, ubiquitination and O-glycosylation (Chen et al., 2008; Wang et al., 2009; Chen et al., 2011; Zhao et al., 2012; Tung, 2013; Zhang et al., 2013).

\section{Feature Normalization}

All the features are normalized by the following formula

$$
\mathrm{X}_{k}^{n}=\frac{x_{k}^{n}-\min _{m}\left\{x_{k}^{m}\right\}}{\max _{m}\left\{x_{k}^{m}\right\}-\min _{m}\left\{x_{k}^{m}\right\}},
$$

where $x_{k}^{n}$ denotes the $\mathrm{k}$-th non-normalized feature of the sample $n$. The normalized feature lies between 0 and 1.

\section{Random Forest}

Random forest by Breiman (2001) is an ensemble learning algorithm which combines decision trees for vote. The random forest is composed mainly of constructing of decision trees and voting over all the decision trees for the given sample. Each decision tree grow out of the new training set drawn with replacement from the training set and with $m<M$ randomly selected features ( $M$ is the total number of sample features). The majority of vote for a sample is the output class for classification. The advantage of Random forests is that it overcome overfitting which occurred in decision trees, and meanwhile produce a limiting value of the generalization error. For more details of random forest, readers can refer to relevant references. Here, we 
use Weka software package (Hall et al., 2009) which realized a wide range of machine learning algorithms using the Java programming language.

\section{CROSS VALIDATION AND METRICS}

We used 3-fold cross validation to examine performance of the proposed method. For 3 -fold cross validation, $n$ training samples are divided into three parts in approximate or equal size. Each part is in turn used as the testing set which is predicted by the trained classifier over the other two parts. Independent test was used to examine generalization ability of the proposed method.

The receiver operating characteristic (ROC) curve was used to assess the predictive performances, which is plotting true positive rate against false positive rate under various threshold. Area under the ROC curve (AUC) was used to compare it, ranging from 0 to 1 . The AUC was 1, meaning the perfect prediction, while the AUC was 0.5, indicating the uninformative classifier.

\section{RESULTS AND DISCUSSION}

To investigate effects of the parameter $N$ (length of amino acid residues in the upstream or the downstream of the butyrylation sites) on the predictive performances, we conducted 3-fold cross validation over the training set. Most approaches for predicting PTM sites generally set $N$ to the interval of 10 to 15 (Hou et al., 2014; Huang et al., 2014; Xu et al., 2015a; Hasan et al., 2016; Jia et al., 2016a; Jia et al., 2016b; Xu et al., 2016; Wang et al., 2017). For example, the iSulf-Cys for predicting s-sulfenylation sites (Xu et al., 2016) adopted a window of 21 residues (i.e., $N=10$ ), while the iSuc-PseOpt (Jia et al., 2016a), a tool for predicting lysine succinylation sites, used $N=15$ amino acid residues of the upstream/downstream of the modified site. Therefore, we tested $N$ only between 10 to 15 . As shown in Figure 2, the ROC curves of 3 -fold cross validation under various $N$ were plotted. The best AUC $(\mathrm{N}=13)$ is 0.92 , while the worst $(N=15)$ is 0.73 . Therefore, we set $\mathrm{N}$ to 13 .

ROC curves of 3 -fold cross validation over the training set for single type of IEE and for single type of CKSAAP features were shown in Figure 3A. The IEE outperformed the CKSAAP and the combination of two. ROC curves of independent test were plotted in Figure 3B. Obviously, the combination performs best, followed by the CKSAAP and then by the IEE feature. The single performance of the IEE feature is best over the training set, but worst over the testing set. The single performance of the CKSAAP is worst over the training set. The combination of IEE and CKSAAP features performs most stable, with 0.92 of AUC over 3 -fold cross validation and 0.80 of AUC over independent test respectively.

\section{Analysis Of Sequence Pattern}

We used the WebLogo program (Crooks et al., 2004) to draw a sequence logo of all the 26 positive samples both from the training and the testing sets, as shown in Figure $\mathbf{4 A}$. The stacks at the positions 13, 25 and 26 is higher, followed by the positions 22, 18 and 11, indicating that these positions would be more evolutionarily conservative. On the contrary, the stacks at the positions $1,7,8$ and 19 is lower, implying these positions would be less conservative. The symbols A (alanine) at the positions $3,6,12,13$, and $26, \mathrm{~K}$ (lysine) at the positions 5,10 , 18,21 and $24, \mathrm{G}$ (glycine) at the positions 9, 11 and 22 , and $\mathrm{R}$ (arginine) at the position 25 are higher at respective stack, indicating that these amino acids alanine, lysine, glycine and arginine would appear more frequently at these corresponding positions. The two-sample sequence logo was plotted using a webbased software (Vacic et al., 2006) http://www.twosamplelogo.org/ index.html. The positive samples were 26 non-redundant fragments containing butyrylation sites, while the negative ones were 36 fragments, 62 in total. In comparison to previous singlesample sequence logo, the two-sample logo more intuitively exhibited statistically significant differential residues between two classes. As shown in Figure 4B , the symbols $\mathrm{K}$ at these positions 21 and 22, A at these positions 3, 13,19 and 20, P (proline) at the position 2, M (methionine) at the position 9, Q (glutamine) at the position $10, \mathrm{~S}$ (serine) at the position $12, \mathrm{G}$ and $\mathrm{R}$ at the position 25 , were enriched in the butyrylation fragments, while $G$ at the position $1, \mathrm{~A}$ at the position $9, \mathrm{~K}$ at the position $13, \mathrm{~S}$ at the position 22, $\mathrm{V}$ (valine) at the positions 15 and 25, and $\mathrm{T}$ (threonine) at the position 25 were depleted. Combining the information from Figures 4A, B, we speculated that alanine at the position 3 and 13, lysine at the position 21 and arginine at the position 25 would be associated with histone butyrylation.

\section{Analysis of Information Entropy Feature}

As shown in Figure 5, we calculated information entropies of all the used positive and the negative samples in the experiment using the equations (1) and (2). Regardless of amino acid or position, information entropies of butyrylation wholly are less than those of non-butyrylation, indicating that the distribution of amino acid followed more a rule in the butyrylation than at random. The information entropies of $\mathrm{C}$ (cysteine) and $\mathrm{W}$ (tryptophan) are near or equal to zero (Figure 5A), implying that two types of amino acid would occur in a fixed way not at random. The information entropies of $\mathrm{F}$ (phenylalanine) and $\mathrm{N}$ (asparagine) are much less in the butyrylation than in the nonbutyrylation, indicating that phenylalanine and asparagine would play a role in the butyrylation. Information entropies of G, P, M and R in the positive sample is approximately equal or more than those in the negative samples, respectively. This indicated non-difference of these amino acids between butyrylation and non-butyrylation. The information entropies of position in the butyrylation is less than those in the nonbutyrylation exception the position 14 (Figure 5B), indicating that amino acid distribution in the butyrylation would follow more rules than at random.

\section{Analysis of CKSAAP Feature}

We calculated pairs of amino acid separated by up to one residue. Namely, amino acid pair might be of such form as $\alpha \beta$ and $\alpha \Delta \beta$, where $\Delta$ represent an amino acid. Figure $\mathbf{6}$ shows frequency of pair of amino acid. Obviously, distribution of amino acid pairs in 


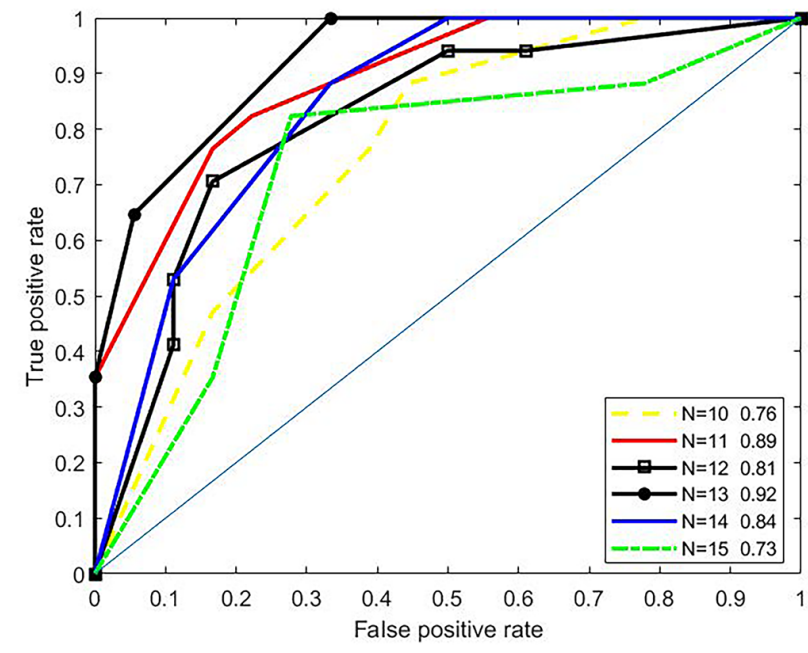

FIGURE 2 | ROC curves under various parameter $N$.

the butyrylation differs largely from that in the non-butyrylation. The butyrylation focuses mainly on these amino acid pairs of DN, GG, GK, KA, KD, KL, KP, KS, KV, PE, RH, RN, VY, XM and $\mathrm{XX}$, while the non-butyrylation on GK, KA, KK and XX.

\section{Analysis of Function for Histone Butyrylation}

We used the PANTHER classification system (Mi et al., 2013) (http://www.pantherdb.org/) for functional analysis of histone butyrylation. Both statistical over-representation tests of Homo sapiens butyrylation histones against the whole $H$. sapiens genes and of Mus musculus butyrylation histones against the whole $M$. musculus genes were performed. The significantly over- represented GO terms $(P<0.05)$ for biological process, molecular function and cellular composition are listed in Supplementary Table 2-7. It is obviously observed that all GO terms of M. musculus butyrylated histones appeared in the $H$. sapiens histones, except cytosol (GO:0005829) which is defined as the part of the cytoplasm which does not contain organelles but contain such particulate matter as protein complexes. However, some GO terms of $H$. sapiens butyrylated histones fail to fall into the set of GO terms of $M$. musculus histones. For example, in terms of molecular function, STAT family protein binding (GO:0097677), RNA polymerase II core promoter sequence-specific DNA binding (GO:0000979), core promoter sequence-specific DNA binding (GO:0001046), core promoter binding (GO:0001047), chromatin binding (GO:0003682), protein-containing complex binding (GO:0044877), protein binding (GO:0005515) and binding (GO:0005488) are significant over-represented GO terms in $H$. sapiens butyrylation histone, not in $M$. musculus histones. The difference of the first four molecular functions between two species would be caused by the small-sample question. The number of studied M. musculus butyrylated histones is 17 , less than the number of $H$. sapiens histones. The term GO:0097677 appeared two times, and these three terms GO:0000979, GO:0001046 and GO:0001047 appeared three times in these 30 butyrylated $H$. sapiens histones, while they would likely appear less than two times in these 17 butyrylated M. musculus histones. Only functions appearing two times or more would be statistically analyzed. Therefore, these four molecular functions could not separate $H$. sapiens from $M$. musculus histones. GO:0044877 appeared 10 times, GO:0003682 11 times, GO:0005515 29 times, while GO:0005488 appeared 30 times in the $H$. sapiens histone. It is rational to infer occurring more than two times in $17 \mathrm{M}$. musculus butyrylation histones, but they were not significant over-represented GO terms. This indicated that
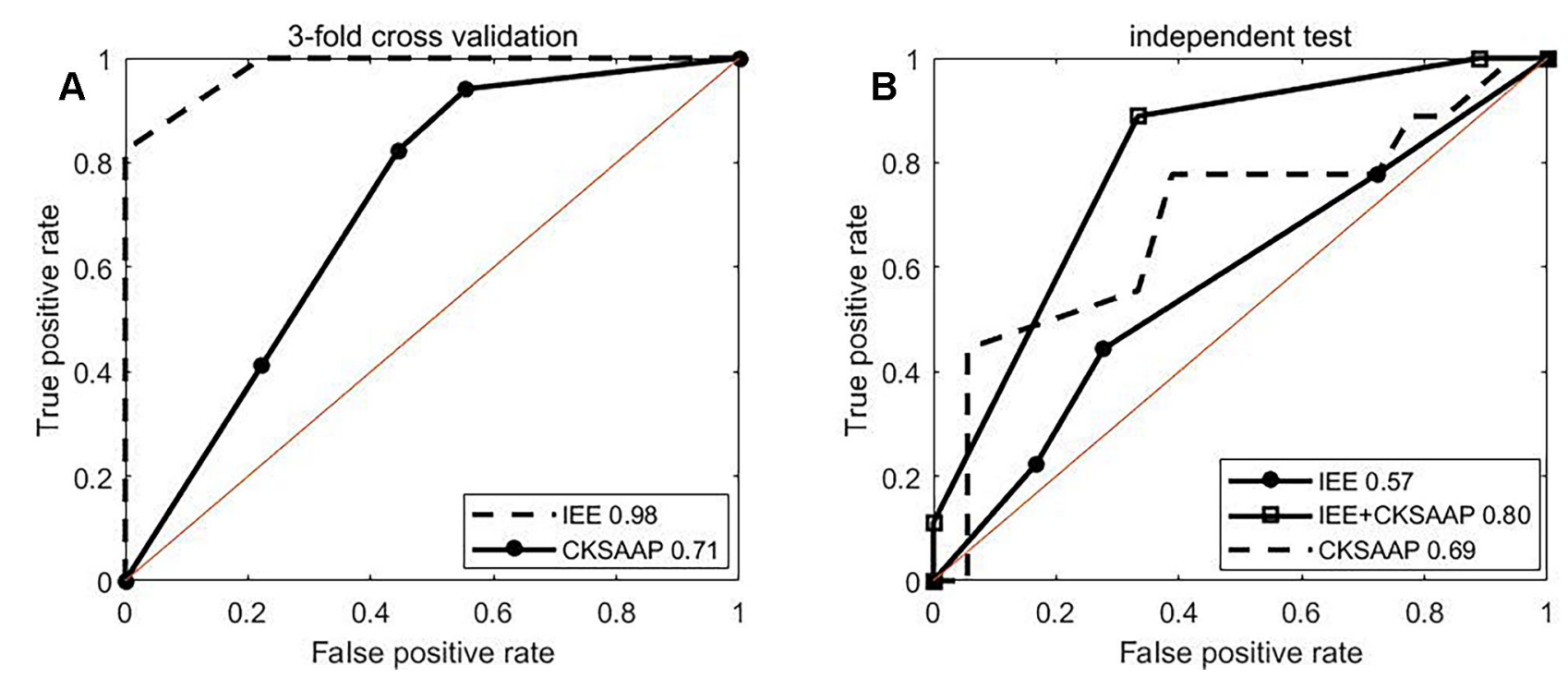

FIGURE 3 | ROC curves. (A, B) depict ROC curves of 3-fold cross validation and independent test, respectively. 


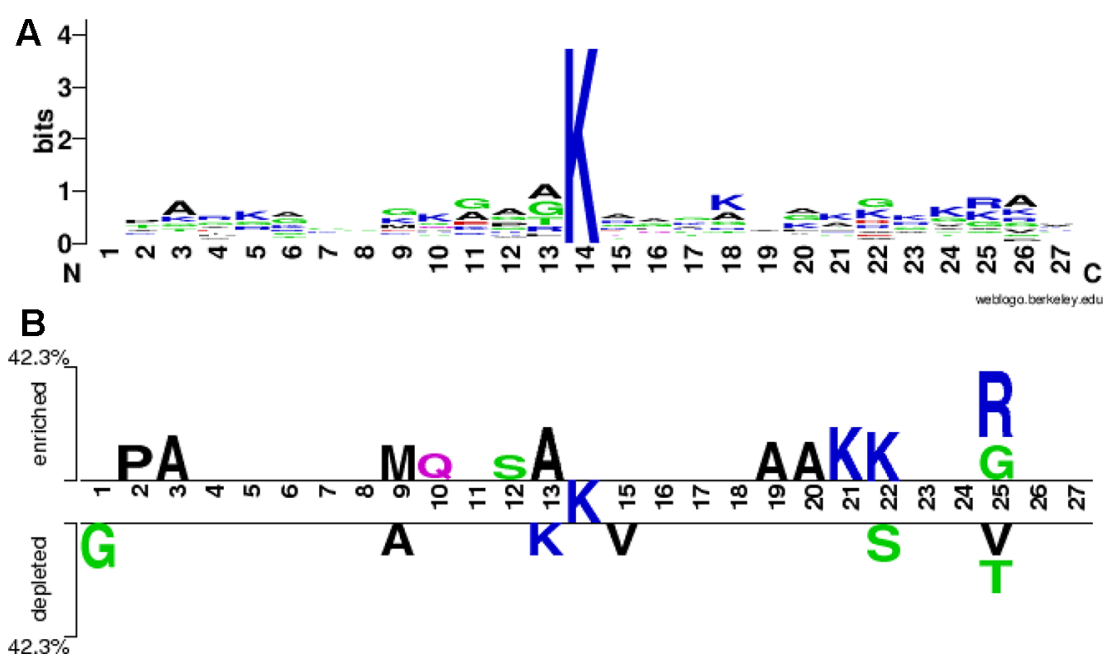

FIGURE 4 | Sequence logo. (A) is sequence logo of all the positive samples and (B) is sequence logo of all the positive and the negative samples.
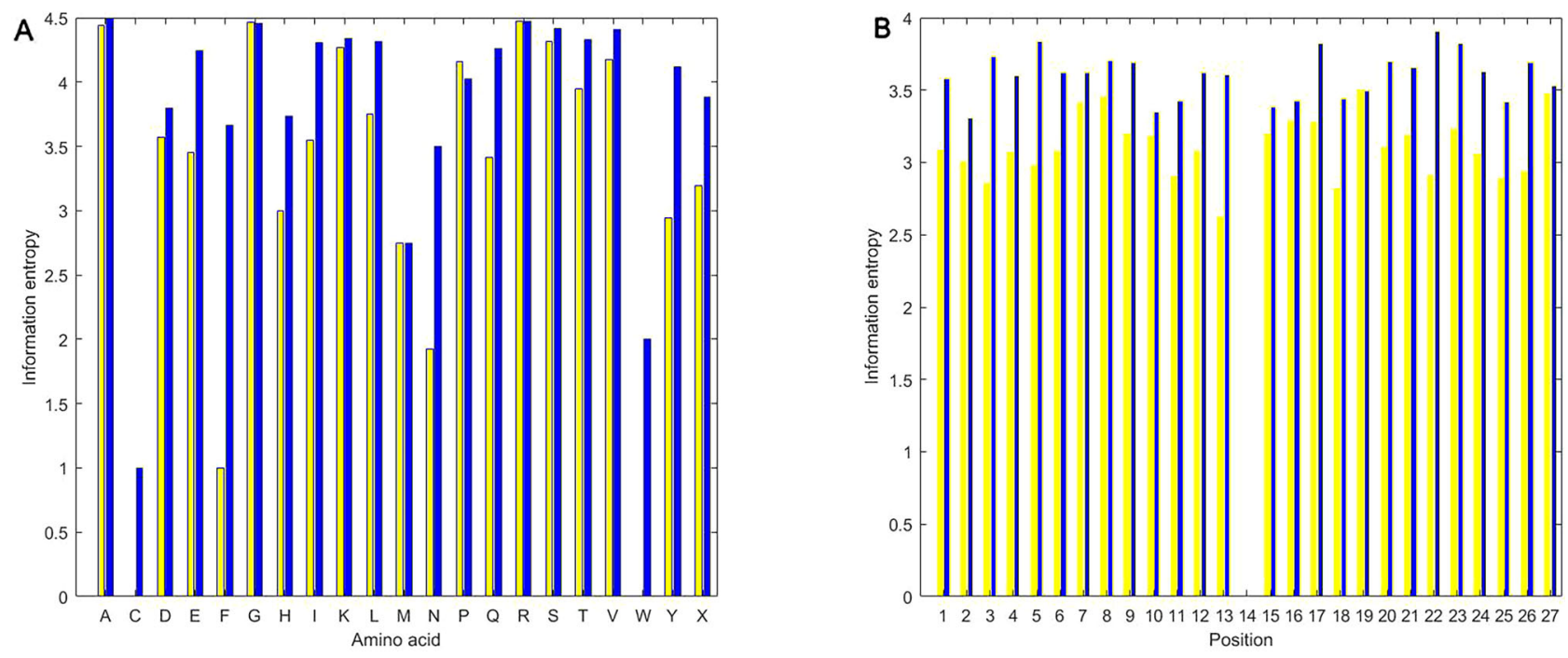

FIGURE 5 | Information entropies. (A) represents information entropies of PIEA and NIEA. (B) represents information entropies of PIEP and NIEP.

these later four molecular functions were enriched only in the $H$. sapiens, not in all the species.

Table 1 listed the most significant five GO terms of molecular function, biological process and cellular component in the butyrylated $H$. sapiens histone which all belonged to the set of the over-represent GO terms in the M. musculus histones respectively. These three terms GO:0003677 (DNA binding), GO:0003676 (nucleic acid binding) and GO:0031492 (nucleosomal DNA binding) are defined as interacting selectively and non-covalently with DNA, with any nucleic acid and with the DNA portion of a nucleosome, respectively. GO:0046982 (protein heterodimerization activity) is defined as interacting selectively and non-covalently with a non-identical protein to form a heterodimer, whose relationship with GO:0046983 (protein dimerization activity) is "is a". All the five terms belongs to the ancestor GO:0005488 (binding) via the "is a" relationship, implying that butyrylation histones could bind other molecules such as DNA, nucleic acid or protein. GO:0006334 (nucleosome assembly) is defined as the aggregation, arrangement and bonding together of a nucleosome, the beadlike structural units of eukaryotic chromatin composed of histones and DNA, which is of "is a" relationship with GO:0034728 (nucleosome organization) and of "part of " relationship with GO:0031497 (chromatin assembly). The term GO:0031497 is of "part of" relationship with GO:0006323 (DNA packaging) and of "is a" relationship with 

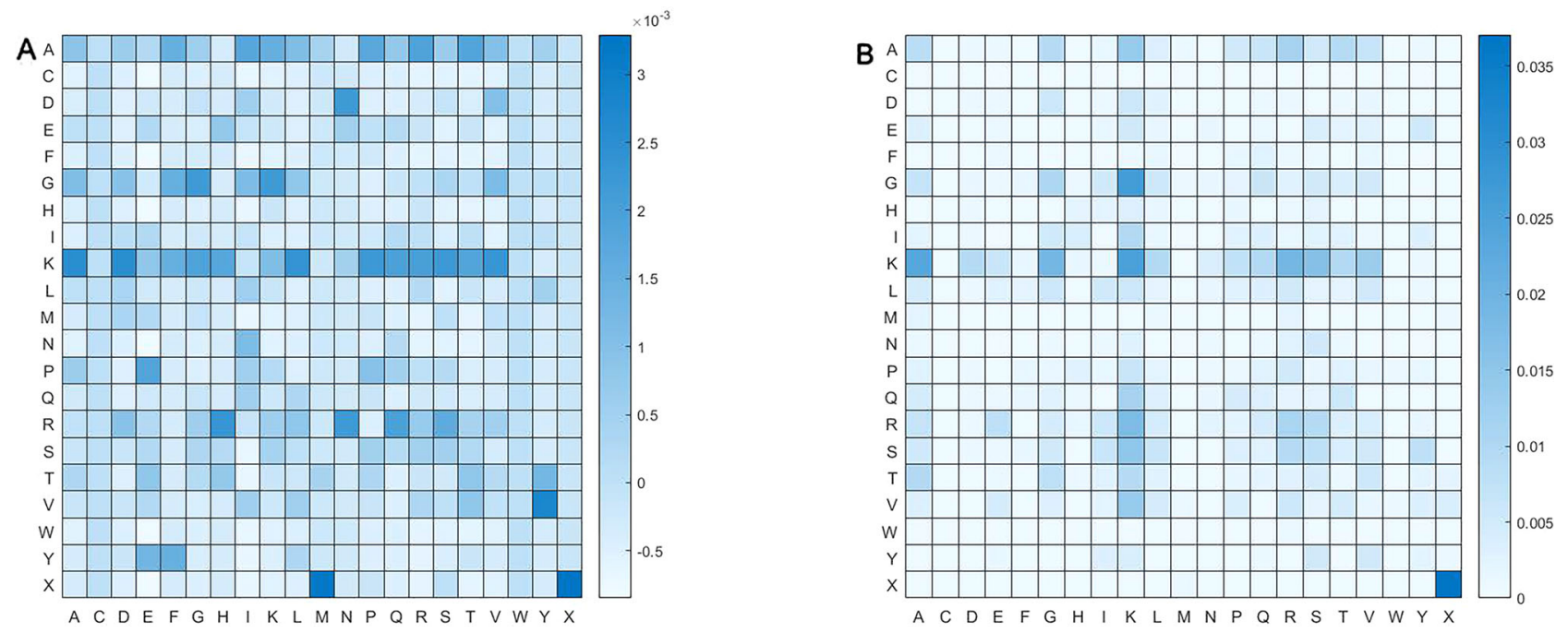

FIGURE 6 | Heatmap of amino acid pair. (A) represents heatmap of all the positive samples and (B) heatmap of all the negative samples.

TABLE 1 | Most significant five GO terms of molecular function, biological process and cellular component for Homo sapiens.

Molecular function

Protein heterodimerization activity (GO:0046982)

DNA binding (GO:0003677)

Protein dimerization activity (GO:0046983)

Nucleic acid binding (GO:0003676)

Nucleosomal DNA binding (GO:0031492)
Biological process

Nucleosome assembly (GO:0006334)

Chromatin assembly (GO:0031497)

Chromatin assembly or disassembly (GO:0006333)

Nucleosome organization (GO:0034728)

DNA packaging (GO:0006323)
Cellular component

Nucleosome (GO:0000786)

DNA packaging complex (GO:0044815)

Protein-DNA complex (GO:0032993)

Chromatin (GO:0000785)

Chromosome (GO:0005694)
GO:0006333 (chromatin assembly or disassembly). These five terms finally are traced up to two terms: GO:0016043 (cellular component organization) and GO:0044085 (cellular component biogenesis), indicating that butyrylation histones might be associated with these processes of biosynthesis, assembly, arrangement or disassembly. The term GO:0000786 (nucleosome) refers to a complex consisting of DNA wound around a multi-subunit core and associated proteins, which forms the primary packing unit of DNA into higher order structures. The term GO:0000786 is of "is a" relationships both with the term GO:0044815 (DNA packaging complex) and with GO:0032993 (protein-DNA complex) and is of "part of" relationship with the term GO:0000785 (chromatin) which is of part of relationship with the term GO:0005694 (chromosome). These results indicate that butyrylation histone might be located in a complex composed of DNA, proteins, etc.

We used the David (Database for Annotation, Visualization and Integrated Discovery) (Huang da et al., 2009a; Huang da et al., 2009b) to explore biological pathways in which the butyrylated histones are potential to be involved. The David is one of most popular tool for enrichment analysis of gene function, currently including over 40 annotation categories, such as ordinary GO terms, protein functional domains, biopathways, etc. The backgrounds for H. sapiens and M. musculus butyrylation histones were respectively the whole H. sapiens and the whole M. musculus genes. The statistically significant Kyoto Encyclopedia of Genes and Genomes (KEGG) pathways ( $P$ value $<0.01)$ are systemic lupus erythematosus, alcoholism, viral carcinogenesis and transcriptional misregulation in cancer, whether for $H$. sapiens or for M. musculus genes, indicating that histone butyrylation is involved in similar bio-pathway.

\section{CONCLUSION}

Histone butyrylation is a newly discovered PTM, whose mechanism remains unknown. In this paper, we presented an approach based on information entropy and machine learning for identifying histone butyrylation sites. To the best of our knowledge, this is the first computational method for identifying histone butyrylation sites. By comparing sequences, IEE and CKSAAP between butyrylation and non-butyrylation, we found some specific characteristics implying potential and hidden pattern of histone butyrylation. The statistical test suggests that the butyrylation histone might be of binding with other molecules, be associated with the processes of biosynthesis, assembly, arrangement or disassembly, be located in the 
complex of DNA, protein, etc, and be involved in the such pathway as systemic lupus erythematosus, alcoholism, viral carcinogenesis and transcriptional misregulation in cancer.

\section{DATA AVAILABILITY STATEMENT}

Butyrylated proteins were retrieved by searching both the Uniprot database(UniProt Consortium, 2018): https:/www. uniprot.org/ and the Protein Lysine Modifications Database: http://plmd.biocuckoo.org/_ (P68432; P62804; P62807;P02294; Q6DN03; Q3SZB8; O75367; P0C0S5; P16104; Q02539; Q75WM6; Q8IZA3; Q92522).

\section{AUTHOR CONTRIBUTIONS}

GH, YZ and Z-GY conceived the method. GH and YZ collected data. $\mathrm{GH}$ and $\mathrm{YZ}$ performed the experiment. GH, YZ, Y-QW, G-

\section{REFERENCES}

Basu, A., Rose, K. L., Zhang, J., Beavis, R. C., Ueberheide, B., Garcia, B. A., et al. (2009). Proteome-wide prediction of acetylation substrates. Proc. Natl. Acad. Sci. U. S. A. 106 (33), 13785-13790. doi: 10.1073/pnas.0906801106

Blom, N., Sicheritz-Ponten, T., Gupta, R., Gammeltoft, S., and Brunak, S. (2004). Prediction of post-translational glycosylation and phosphorylation of proteins from the amino acid sequence. Proteomics 4 (6), 1633-1649. doi: 10.1002/ pmic. 200300771

Breiman, L. (2001). Random forests. Mach. Learn. 45 (1), 5-32. doi: 10.1023/ A:1010933404324

Cai, Y., Huang, T., Hu, L., Shi, X., Xie, L., and Li, Y. (2012). Prediction of lysine ubiquitination with mRMR feature selection and analysis. Amino Acids 42 (4), 1387-1395. doi: 10.1007/s00726-011-0835-0

Caragea, C., Sinapov, J., Silvescu, A., Dobbs, D., and Honavar, V. (2007). Glycosylation site prediction using ensembles of support vector machine classifiers. BMC Bioinf. 8, 438. doi: 10.1186/1471-2105-8-438

Chen, H., Xue, Y., Huang, N., Yao, X., and Sun, Z. (2006). MeMo: a web tool for prediction of protein methylation modifications. Nucleic Acids Res. 34 (Web Server issue), W249-W253. doi: 10.1093/nar/gkl233

Chen, Y., Sprung, R., Tang, Y., Ball, H., Sangras, B., Kim, S. C., et al. (2007). Lysine propionylation and butyrylation are novel post-translational modifications in histones. Mol. Cell Proteomics 6 (5), 812-819. doi: 10.1074/mcp.M700021MCP200

Chen, Y. Z., Tang, Y. R., Sheng, Z. Y., and Zhang, Z. (2008). Prediction of mucintype O-glycosylation sites in mammalian proteins using the composition of k-spaced amino acid pairs. BMC Bioinf. 9, 101. doi: 10.1186/1471-2105-9101

Chen, Z., Chen, Y. Z., Wang, X. F., Wang, C., Yan, R. X., and Zhang, Z. (2011). Prediction of ubiquitination sites by using the composition of k-spaced amino acid pairs. PloS One 6 (7), e22930. doi: 10.1371/journal.pone.0022930

Choudhary, C., Weinert, B. T., Nishida, Y., Verdin, E., and Mann, M. (2014). The growing landscape of lysine acetylation links metabolism and cell signalling. Nat. Rev. Mol. Cell Biol. 15 (8), 536-550. doi: 10.1038/nrm3841

Chuang, G.-Y., Boyington, J. C., Joyce, M. G., Zhu, J., Nabel, G. J., Kwong, P. D., et al. (2012). Computational prediction of N-linked glycosylation incorporating structural properties and patterns. Bioinformatics 28 (17), 2249-2255. doi: 10.1093/bioinformatics/bts426

Crooks, G. E., Hon, G., Chandonia, J.-M., and Brenner, S. E. (2004). WebLogo: a sequence logo generator. Genome Res. 14 (6), 1188-1190. doi: 10.1101/ gr.849004
$\mathrm{SH}$ and Z-GY analyzed the results and wrote the manuscript. All the authors read the manuscript and approved the final manuscript.

\section{FUNDING}

This work is supported by National Natural Science Foundation of China $(61672356,11871061)$, by Scientific Research Fund of Hunan Provincial Education Department (18A394), and by the open project of Hunan Key Laboratory for Computation and Simulation in Science and Engineering (2019LCESE03).

\section{SUPPLEMENTARY MATERIAL}

The Supplementary Material for this article can be found online at: https://www.frontiersin.org/articles/10.3389/fgene.2019.01325/ full\#supplementary-material

Gnad, F., Ren, S., Choudhary, C., Cox, J., and Mann, M. (2010). Predicting posttranslational lysine acetylation using support vector machines. Bioinformatics 26 (13), 1666-1668. doi: 10.1093/bioinformatics/btq260

Goudarzi, A., Zhang, D., Huang, H., Barral, S., Kwon, O. K., Qi, S., et al. (2016). Dynamic competing histone $\mathrm{H} 4 \mathrm{~K} 5 \mathrm{~K} 8$ acetylation and butyrylation are hallmarks of highly active gene promoters. Mol. Cell 62 (2), 169-180. doi: 10.1016/j.molcel.2016.03.014

Hall, M., Frank, E., Holmes, G., Pfahringer, B., Reutemann, P., and Witten, I. H. (2009). The WEKA data mining software: an update. ACM SIGKDD Explor. Newsl. 11 (1), 10-18. doi: 10.1145/1656274.1656278

Hamamoto, R., Saloura, V., and Nakamura, Y. (2015). Critical roles of non-histone protein lysine methylation in human tumorigenesis. Nat. Rev. Cancer 15 (2), 110-124. doi: $10.1038 / \mathrm{nrc} 3884$

Hamby, S. E., and Hirst, J. D. (2008). Prediction of glycosylation sites using random forests. BMC Bioinf. 9, 500. doi: 10.1186/1471-2105-9-500

Hansen, J. E., Lund, O., Engelbrecht, J., Bohr, H., and Nielsen, J. O. (1995). Prediction of O-glycosylation of mammalian proteins: specificity patterns of UDP-GalNAc: polypeptide N-acetylgalactosaminyltransferase. Biochem. J. 308, 801-813. doi: 10.1042/bj3080801

Hansen, J. E., Lund, O., Tolstrup, N., Gooley, A. A., Williams, K. L., and Brunak, S. (1998). NetOglyc: prediction of mucin type O-glycosylation sites based on sequence context and surface accessibility. Glycoconj. J. 15 (2), 115-130. doi: 10.1023/a:1006960004440

Hasan, M. M., Yang, S., Zhou, Y., and Mollah, M. N. (2016). SuccinSite: a computational tool for the prediction of protein succinylation sites by exploiting the amino acid patterns and properties. Mol. Biosyst. 12 (3), 786795. doi: $10.1039 / \mathrm{c} 5 \mathrm{mb} 00853 \mathrm{k}$

Hou, T., Zheng, G., Zhang, P., Jia, J., Li, J., Xie, L., et al. (2014). LAceP: lysine acetylation site prediction using logistic regression classifiers. PloS One 9 (2), e89575. doi: 10.1371/journal.pone.0089575

Huang da, W., Sherman, B. T., and Lempicki, R. A. (2009a). Bioinformatics enrichment tools: paths toward the comprehensive functional analysis of large gene lists. Nucleic Acids Res. 37 (1), 1-13. doi: 10.1093/nar/gkn923

Huang da, W., Sherman, B. T., and Lempicki, R. A. (2009b). Systematic and integrative analysis of large gene lists using DAVID bioinformatics resources. Nat. Protoc. 4 (1), 44-57. doi: 10.1038/nprot.2008.211

Huang, Y., Niu, B., Gao, Y., Fu, L., and Li, W. (2010). CD-HIT Suite: a web server for clustering and comparing biological sequences. Bioinformatics 26 (5), 680 682. doi: 10.1093/bioinformatics/btq003

Huang, G., Lu, L., Feng, K., Zhao, J., Zhang, Y., Xu, Y., et al. (2014). Prediction of S-nitrosylation modification sites based on kernel sparse representation 
classification and mRMR algorithm. BioMed. Res. Int. 2014, 438341. doi: $10.1155 / 2014 / 438341$

Jia, J., Liu, Z., Xiao, X., Liu, B., and Chou, K. C. (2016a). iSuc-PseOpt: identifying lysine succinylation sites in proteins by incorporating sequence-coupling effects into pseudo components and optimizing imbalanced training dataset. Anal. Biochem. 497, 48-56. doi: 10.1016/j.ab.2015.12.009

Jia, J., Liu, Z., Xiao, X., Liu, B., and Chou, K. C. (2016b). pSuc-Lys: predict lysine succinylation sites in proteins with PseAAC and ensemble random forest approach. J. Theor. Biol. 394, 223-230. doi: 10.1016/j.jtbi.2016.01.020

Kiemer, L., Bendtsen, J. D., and Blom, N. (2005). NetAcet: prediction of Nterminal acetylation sites. Bioinformatics 21 (7), 1269-1270. doi: 10.1093/ bioinformatics/bti130

Li, S., Liu, B., Zeng, R., Cai, Y., and Li, Y. (2006). Predicting O-glycosylation sites in mammalian proteins by using SVMs. Comput. Biol. Chem. 30 (3), 203-208. doi: 10.1016/j.compbiolchem.2006.02.002

Liu, Z., Cao, J., Ma, Q., Gao, X., Ren, J., and Xue, Y. (2011). GPS-YNO2: computational prediction of tyrosine nitration sites in proteins. Mol. Biosyst. 7 (4), 1197-1204. doi: $10.1039 / \mathrm{c} 0 \mathrm{mb} 00279 \mathrm{~h}$

Lu, Y., Xu, Q., Liu, Y., Yu, Y., Cheng, Z. Y., Zhao, Y., et al. (2018). Dynamics and functional interplay of histone lysine butyrylation, crotonylation, and acetylation in rice under starvation and submergence. Genome Biol. 19 (1), 144. doi: 10.1186/s13059-018-1533-y

Mi, H., Muruganujan, A., Casagrande, J. T., and Thomas, P. D. (2013). Large-scale gene function analysis with the PANTHER classification system. Nat. Protoc. 8 (8), 1551-1566. doi: 10.1038/nprot.2013.092

Qiu, W. R., Xiao, X., Lin, W. Z., and Chou, K. C. (2014). iMethyl-PseAAC: identification of protein methylation sites via a pseudo amino acid composition approach. BioMed. Res. Int. 2014, 947416. doi: 10.1155/2014/947416

Sasaki, K., Nagamine, N., and Sakakibara, Y. (2009). Support vector machine prediction of $\mathrm{N}$-and O-glycosylation sites using whole sequence information and subcellular localization. IPSJ Trans. Bioinf. 2, 25-35. doi: 10.2197/ipsitbio.2.25

Shao, J., Xu, D., Tsai, S. N., Wang, Y., and Ngai, S. M. (2009). Computational identification of protein methylation sites through bi-profile Bayes feature extraction. PloS One 4 (3), e4920. doi: 10.1371/journal.pone.0004920

Shi, S. P., Qiu, J. D., Sun, X. Y., Suo, S. B., Huang, S. Y., and Liang, R. P. (2012a). PLMLA: prediction of lysine methylation and lysine acetylation by combining multiple features. Mol. Biosyst. 8 (5), 1520-1527. doi: 10.1039/c2mb05502c

Shi, S. P., Qiu, J. D., Sun, X. Y., Suo, S. B., Huang, S. Y., and Liang, R. P. (2012b). PMeS: prediction of methylation sites based on enhanced feature encoding scheme. PloS One 7 (6), e38772. doi: 10.1371/journal.pone.0038772

Shi, Y., Guo, Y., Hu, Y., and Li, M. (2015). Position-specific prediction of methylation sites from sequence conservation based on information theory. Sci. Rep. 5, 12403. doi: 10.1038/srep 12403

Shien, D. M., Lee, T. Y., Chang, W. C., Hsu, J. B., Horng, J. T., Hsu, P. C., et al. (2009). Incorporating structural characteristics for identification of protein methylation sites. J. Comput. Chem. 30 (9), 1532-1543. doi: 10.1002/jcc.21232

Tung, C. W. (2013). Prediction of pupylation sites using the composition of kspaced amino acid pairs. J. Theor. Biol. 336, 11-17. doi: 10.1016/ j.jtbi.2013.07.009

UniProt Consortium, T. (2018). UniProt: the universal protein knowledgebase. Nucleic Acids Res. 46 (5), 2699. doi: 10.1093/nar/gky092

Vacic, V., Iakoucheva, L. M., and Radivojac, P. (2006). Two Sample Logo: a graphical representation of the differences between two sets of sequence alignments. Bioinformatics 22 (12), 1536-1537. doi: 10.1093/bioinformatics/btll51

Wang, X. B., Wu, L. Y., Wang, Y. C., and Deng, N. Y. (2009). Prediction of palmitoylation sites using the composition of k-spaced amino acid pairs. Protein Eng. Des. Sel. 22 (11), 707-712. doi: 10.1093/protein/gzp055

Wang, L. N., Shi, S. P., Xu, H. D., Wen, P. P., and Qiu, J. D. (2017). Computational prediction of species-specific malonylation sites via enhanced characteristic strategy. Bioinformatics 33 (10), 1457-1463. doi: 10.1093/bioinformatics/ btw755

Wei, L., Xing, P., Shi, G., Ji, Z. L., and Zou, Q. (2018). Fast prediction of protein methylation sites using a sequence-based feature selection technique. IEEE/ ACM Trans. Comput. Biol. Bioinf. PP (99), 1-1. doi: 10.1109/ TCBB.2017.2670558

Xu, J., He, Y., Qiang, B., Yuan, J., Peng, X., and Pan, X. M. (2008). A novel method for high accuracy sumoylation site prediction from protein sequences. $B M C$ Bioinf. 9, 8. doi: 10.1186/1471-2105-9-8
Xu, Y., Wang, X.-B., Ding, J., Wu, L.-Y., and Deng, N.-Y. (2010). Lysine acetylation sites prediction using an ensemble of support vector machine classifiers. J. Theor. Biol. 264 (1), 130-135. doi: 10.1016/j.jtbi.2010.01.013

Xu, Y., Ding, J., Huang, Q., and Deng, N.-Y. (2013). Prediction of protein methylation sites using conditional random field. Protein Pept. Lett. 20 (1), 71-77. doi: 10.2174/092986613804096865

Xu, G., Wang, J., Wu, Z., Qian, L., Dai, L., Wan, X., et al. (2014). SAHA regulates histone acetylation, Butyrylation, and protein expression in neuroblastoma. J. Proteome Res. 13 (10), 4211-4219. doi: 10.1021/pr500497e

Xu, H. D., Shi, S. P., Wen, P. P., and Qiu, J. D. (2015a). SuccFind: a novel succinylation sites online prediction tool via enhanced characteristic strategy. Bioinformatics 31 (23), 3748-3750. doi: 10.1093/bioinformatics/btv439

Xu, Y., Ding, Y. X., Ding, J., Lei, Y. H., Wu, L. Y., and Deng, N. Y. (2015b). iSucPseAAC: predicting lysine succinylation in proteins by incorporating peptide position-specific propensity. Sci. Rep. 5, 10184. doi: 10.1038/srep10184

Xu, Y., Ding, J., and Wu, L. Y. (2016). iSulf-Cys: prediction of S-sulfenylation sites in proteins with physicochemical properties of amino acids. PloS One 11 (4), e0154237. doi: 10.1371/journal.pone.0154237

Xu, H., Zhou, J., Lin, S., Deng, W., Zhang, Y., and Xue, Y. (2017). PLMD: an updated data resource of protein lysine modifications. J. Genet. Genomics 44 (5), 243-250. doi: 10.1016/j.jgg.2017.03.007

Xu, J. Y., Xu, Z., Liu, X., Tan, M., and Ye, B. C. (2018). Protein acetylation and butyrylation regulate the phenotype and metabolic shifts of the endosporeforming Clostridium acetobutylicum. Mol. Cell Proteomics 17 (6), 1156-1169. doi: 10.1074/mcp.RA117.000372

Xue, Y., Chen, H., Jin, C., Sun, Z., and Yao, X. (2006). NBA-Palm: prediction of palmitoylation site implemented in naive Bayes algorithm. BMC Bioinf. 7, 458. doi: 10.1186/1471-2105-7-458

Zhang, K., Chen, Y., Zhang, Z., and Zhao, Y. (2008). Identification and verification of lysine propionylation and butyrylation in yeast core histones using PTMap Software. J. Proteome Res. 8 (2), 900-906. doi: 10.1021/pr8005155

Zhang, N., Li, B.-Q., Gao, S., Ruan, J.-S., and Cai, Y.-D. (2012). Computational prediction and analysis of protein $\gamma$-carboxylation sites based on a random forest method. Mol. Biosyst. 8 (11), 2946-2955. doi: 10.1039/c2mb25185j

Zhang, W., Xu, X., Yin, M., Luo, N., Zhang, J., and Wang, J. (2013). Prediction of methylation sites using the composition of k-spaced amino acid pairs. Protein Pept. Lett. 20 (8), 911-917. doi: 10.2174/0929866511320080008

Zhang, Y., Tang, L., Zou, H., Yang, Q., Yu, X., Jiang, J., et al. (2015). Identifying protein arginine methylation sites using global features of protein sequence coupled with support vector machine optimized by particle swarm optimization algorithm. Chemom. Intell. Lab. Syst. 146, 102-107. doi: 10.1016/j.chemolab.2015.05.011

Zhao, X., Zhang, W., Xu, X., Ma, Z., and Yin, M. (2012). Prediction of protein phosphorylation sites by using the composition of k-spaced amino acid pairs. PloS One 7 (10), e46302. doi: 10.1371/journal.pone.0046302

Zhao, X., Dai, J., Ning, Q., Ma, Z., Yin, M., and Sun, P. (2013). Position-specific analysis and prediction of protein pupylation sites based on multiple features. BioMed. Res. Int. 2013, 109549. doi: 10.1155/2013/109549

Zhou, F. F., Xue, Y., Chen, G. L., and Yao, X. (2004). GPS: a novel group-based phosphorylation predicting and scoring method. Biochem. Biophys. Res. Commun. 325 (4), 1443-1448. doi: 10.1016/j.bbrc.2004.11.001

Zhou, F., Xue, Y., Yao, X., and Xu, Y. (2006). CSS-Palm: palmitoylation site prediction with a clustering and scoring strategy (CSS). Bioinformatics 22 (7), 894-896. doi: 10.1093/bioinformatics/btl013

Zhou, Y., Huang, T., Huang, G., Zhang, N., Kong, X., and Cai, Y.-D. (2016). Prediction of protein $\mathrm{N}$-formylation and comparison with $\mathrm{N}$-acetylation based on a feature selection method. Neurocomputing 217, 53-62. doi: 10.1016/j.neucom.2015.10.148

Conflict of Interest: The authors declare that the research was conducted in the absence of any commercial or financial relationships that could be construed as a potential conflict of interest.

Copyright () 2020 Huang, Zheng, Wu, Han and Yu. This is an open-access article distributed under the terms of the Creative Commons Attribution License (CC BY). The use, distribution or reproduction in other forums is permitted, provided the original author(s) and the copyright owner(s) are credited and that the original publication in this journal is cited, in accordance with accepted academic practice. No use, distribution or reproduction is permitted which does not comply with these terms. 